\title{
Highly tunable exchange-only singlet-only qubit in a GaAs triple quantum dot
}

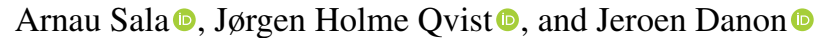 \\ Center for Quantum Spintronics, Department of Physics, Norwegian University of Science and Technology, NO-7491 Trondheim, Norway
}

(Received 22 November 2019; revised manuscript received 26 February 2020; accepted 27 February 2020; published 13 March 2020)

\begin{abstract}
We propose an implementation of a singlet-only spin qubit in a GaAs-based triple quantum dot with a (1, $4,1)$ charge occupation. In the central multielectron dot, the interplay between Coulomb interaction and an out-of-plane magnetic field creates an energy spectrum with a tunable singlet-triplet splitting, which can be exploited to create a six-particle singlet-only qubit with a qubit splitting that can straightforwardly be tuned over tens of $\mu \mathrm{eV}$ by adjusting the external magnetic field. We confirm the full exchange-based electric control of the qubit and demonstrate its superior coherence properties due to its singlet-only nature.
\end{abstract}

DOI: 10.1103/PhysRevResearch.2.012062

Introduction. Semiconductor spin qubits are among the most promising candidates for the physical realization of quantum processors [1,2]. Multispin exchange-only (XO) qubits, in particular, have drawn much attention in recent years since they offer fast qubit manipulation and full electric control [3-10]. However, rapid decoherence of the qubit-due to magnetic noise from randomly fluctuating nuclear spins $[11,12]$, electric noise in the qubit's environment [13-15], electron-phonon coupling [16-18], and other spin-mixing mechanisms [19-22] - still causes the usable operation time of most XO qubits to be too short for scaling up. Besides, the typically small qubit splitting $[4,8]$ hinders the long-distance coupling of XO qubits via, e.g, microwave resonators, where a large qubit splitting is required for fast two-qubit gates [23-25].

There have been several proposals put forward to increase the coherence time of quantum-dot-based XO qubits while retaining their conceptual simplicity and ease of manipulation. Of special interest are (i) proposals to suppress the effects of charge noise and electron-phonon interaction, via a symmetric operation of the qubit or operating at a sweet spot (SS) $[17,26-$ 28], and (ii) proposals to reduce magnetic noise or suppress its effects, either by isotope purification or by constructing decoherence-free qubit subspaces [9,29-32].

In the exchange-only singlet-only (XOSO) spin qubit proposed in Ref. [31], the leading effects of magnetic noise are suppressed by encoding the qubit states in a four-electron singlet-only subspace, while electric noise can be mitigated by operating the system symmetrically at a SS. However, the exceptionally long coherence time of the qubit comes at the cost of an increase in device complexity (a quadruple quantum dot in a $\mathrm{T}$ geometry) and the proposal suffers from the common problem with XO qubits of having a relatively small qubit splitting.

Published by the American Physical Society under the terms of the Creative Commons Attribution 4.0 International license. Further distribution of this work must maintain attribution to the author(s) and the published article's title, journal citation, and DOI.
Here, we propose a GaAs-based implementation of the XOSO qubit that overcomes both drawbacks and, furthermore, has a qubit splitting that is straightforwardly tunable over a large range of energies. The reason why the XOSO qubit of Ref. [31] used a fourth quantum dot is that the qubit splitting scales with the singlet-triplet splitting of the "central" two electrons: Implementing the same qubit in a linear triple dot in a $(1,2,1)$ charge configuration is in principle possible but results in a qubit with a splitting of the order of the orbital level splitting on the central dot $(\sim \mathrm{meV})$, which is too large for practical purposes. In Ref. [32], it was pointed out that one can implement the same qubit in a Si-based triple dot, where the on-site singlet-triplet splitting is typically set by the valley splitting, which can be $20-200 \mu \mathrm{eV}$. The drawback of this proposal is that (i) the magnitude of the valley splitting is hard to control or predict in practice [2] and (ii) uncontrollable phase differences between valley couplings on different dots can severely affect the exchange effects used to define and operate the qubit [33]. Besides, Si can be purified to be almost nuclear spin free, which eradicates the need for a singlet-only qubit [9].

The solution is to tune the triple quantum dot to a $(1,4,1)$ charge configuration and apply an out-of-plane magnetic field. On the central dot, the interplay between the magnetic field and the Coulomb interaction between the electrons results in an energy spectrum with many crossings between levels with different total spin and orbital angular momentum. For the case of four electrons, the ground state changes from a triplet to a singlet character, typically at a moderate field of $\approx 100 \mathrm{mT}$ [34]. Tuning close to this crossing and adding the singly occupied outer dots to the picture yields a XOSO qubit where the singlet-triplet splitting on the central dot, and thus the qubit splitting, can be tuned by adjusting the external magnetic field. This yields a superior GaAs-based XOSO qubit that is not more complicated to create or operate than existing spin qubits and has a qubit splitting that is straightforwardly tunable from zero to tens of $\mu \mathrm{eV}$ [35]. This high degree of tunability could also be beneficial for a Si-based version of this qubit.

Multielectron dot. The single-particle Hamiltonian of an electron labeled $i$ in a two-dimensional planar quantum dot, 
assuming a parabolic confinement and an external magnetic field perpendicular to the plane, is

$$
H_{0}^{(i)}=\frac{\left[\mathbf{p}_{i}+e \mathbf{A}\left(\mathbf{r}_{i}\right)\right]^{2}}{2 m^{*}}+\frac{1}{2} m^{*} \omega_{0}^{2} r_{i}^{2}+\frac{1}{2} g \mu_{\mathrm{B}} B \sigma_{i}^{z},
$$

where $\mathbf{A}(\mathbf{r})=\frac{1}{2} B(x \hat{y}-y \hat{x})$ is the vector potential, $\omega_{0}$ sets the effective radius of the dot in the absence of a magnetic field $\sigma_{0}=\sqrt{\hbar / m^{*} \omega_{0}}, g$ is the $g$ factor of the host material, and $\sigma^{z}$ is the third Pauli matrix. The eigenstates of this Hamiltonian are the Fock-Darwin states

$$
\psi_{n, l, \eta}(\mathbf{r})=\sqrt{\frac{n !}{\pi \sigma^{2}(n+|l|) !}} \rho^{|l|} e^{-\rho^{2} / 2} L_{n}^{|l|}\left(\rho^{2}\right) e^{-i l \theta},
$$

in terms of the dimensionless polar coordinates $\rho=r / \sigma$ and $\theta$. We used $\sigma=\sqrt{\hbar / m^{*} \Omega}$, with $\Omega=\sqrt{\omega_{0}^{2}+\omega_{c}^{2} / 4}$ and $\omega_{c}=$ $e B / m^{*}$, and $L_{a}^{b}(x)$ is the associated Laguerre polynomial. The quantum numbers $n \in \mathbb{N}_{0}, l \in \mathbb{Z}$, and $\eta= \pm 1$ label the radial state, orbital angular momentum, and spin of the electron, respectively. The corresponding eigenenergies are (we will set $\hbar=1$ from now on)

$$
E_{n, l, \eta}=\Omega(2 n+|l|+1)-\frac{1}{2} \omega_{c} l+\frac{1}{4} g \omega_{c} \frac{m^{*}}{m_{e}} \eta .
$$

In order to find the approximate eigenenergies and spin structure of multielectron states in the presence of electron-electron interactions, we follow the method used in Refs. [34,36]; see the Supplemental Material [37] for the details. We create a many-particle basis of antisymmetrized products of single-particle states (2), where we restrict ourselves to the states with $n \leqslant 1$ and $|l| \leqslant 3$, which corresponds to including all single-particle levels up to $\approx 4 \Omega$ at small fields. In the thusly constructed basis, we evaluate all matrix elements of the interaction Hamiltonian

$$
V=\sum_{i<j} \frac{e^{2}}{4 \pi \varepsilon\left|\mathbf{r}_{i}-\mathbf{r}_{j}\right|},
$$

and the eigenstates and eigenenergies of the full many-particle Hamiltonian $H_{1}=\sum_{i} H_{0}^{(i)}+V$ can then be found from numerical diagonalization or, in the weak-interaction limit characterized by $\kappa \equiv e^{2} / 4 \pi \varepsilon \sigma_{0} \omega_{0} \ll 1$, from perturbation theory in $\kappa$. For few particles and not too large $\kappa$ (we consider up to five electrons and $\kappa \leqslant 1.5$ ), the low-energy part of the spectrum of $H_{1}$ will resemble the exact many-particle spectrum fairly accurately $[34,44]$.

In Fig. 1(a), we present typical results for the lowest few levels for the case of four electrons, where we set $\kappa=0.5$ and $g=-0.4$. The dots show the numerically calculated lowest five eigenenergies, where green (blue) dots indicate a state with a four-particle spin singlet (triplet) structure. The three triplet states are labeled $\left|T_{\beta}\right\rangle$ and have the largest weight in the orbital configuration $(0,0)^{2}(0,1)^{1}(0,-1)^{1}$, where $(n, l)^{m}$ means $m$ electrons in the orbital state $(n, l)$ [34]. The three lowest singlet states, labeled $\left|S_{\alpha, \beta, \gamma}\right\rangle$, live mostly in the orbital configurations $(0,0)^{2}(0,1)^{2},(0,0)^{2}(0,1)^{1}(0,-1)^{1}$, and $(0,0)^{2}(0,-1)^{2}$, respectively.

For small $\kappa$, these lowest eigenenergies can also be approximated through perturbation theory in the interaction Hamiltonian $V$. Up to second order in $\kappa$, this yields for the
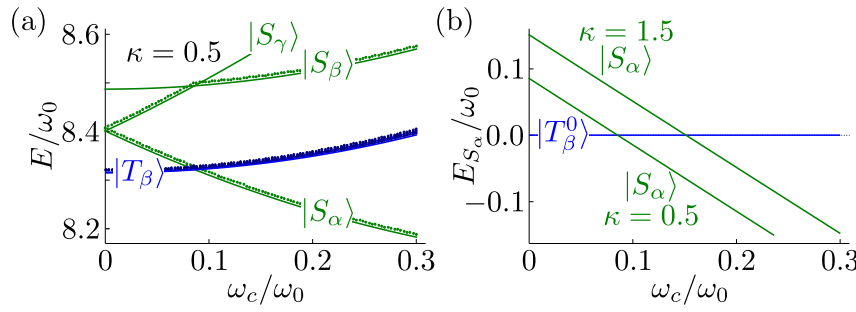

FIG. 1. (a) Field-dependent low-energy part of the spectrum of a four-electron quantum dot with $\kappa=0.5$ and $g=-0.4$. Dots present numerical results and solid lines show the perturbative results of (5). (b) The numerically evaluated energy of the state $\left|S_{\alpha}\right\rangle$ (green lines) relative to $\left|T_{\beta}^{0}\right\rangle$ for two values of $\kappa$.

lowest six states the generic expression

$$
E_{v}=6 \Omega-\frac{L}{2} \omega_{c}+\frac{S}{2} g \omega_{c} \frac{m^{*}}{m_{e}}+c_{1}^{(\nu)} \kappa \sqrt{\Omega \omega_{0}}+c_{2}^{(\nu)} \kappa^{2} \omega_{0},
$$

where $L$ and $S$ denote the total orbital and spin angular momentum along $\hat{z}$ of the four electrons. The coefficients $c_{1,2}^{(v)} \sim 1$ differ per state $|v\rangle$ but can be found explicitly; see Ref. [37] for their exact values. The resulting energies $E_{v}$ are plotted in Fig. 1(a) as solid lines and show good agreement with the numerics. For larger $\kappa$, the perturbation theory breaks down, but the low-energy part of the spectrum is qualitatively the same. This suggests that one can use Eq. (5) to describe the $E_{v}$ if one treats the coefficients $c_{1.2}^{(v)}$ as fit parameters to the numerical data. As illustrated in Ref. [37] for the case $\kappa=1.5$, this still leads to excellent agreement. In Fig. 1(b), we show the numerically evaluated energy of the state $\left|S_{\alpha}\right\rangle$ relative to $\left|T_{\beta}^{0}\right\rangle$ as a function of $\omega_{c}$, for $\kappa=0.5$ and $\kappa=$ 1.5. In both cases, the splitting between $\left|S_{\alpha}\right\rangle$ and $\left|T_{\beta}^{0}\right\rangle$ is to good approximation linear in $\omega_{c}$ in the regime of interest, and the ground state changes from a spin triplet to a singlet around $\omega_{c} / \omega_{0} \sim 0.1$. These two generic features are the key ingredients for our qubit proposal.

Triple-dot six-electron states. We will construct our qubit in two six-electron states hosted in a linear arrangement of three quantum dots with a perpendicular magnetic field applied, such as sketched in Fig. 2(a), where the effective on-site potentials $V_{i}$ and the interdot tunnel couplings $t_{i j}$ can be controlled through nearby gate electrodes, as schematically (a)

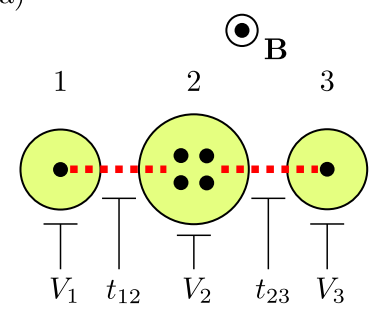

(b)

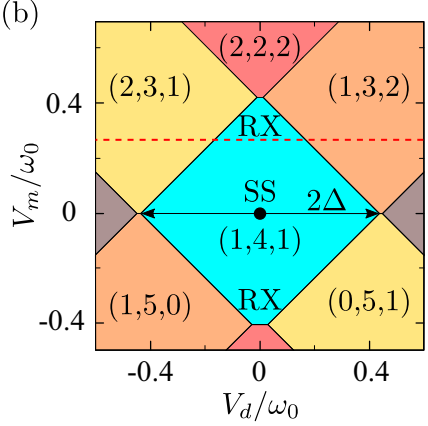

FIG. 2. (a) Sketch of the linear triple-dot setup in a $(1,4,1)$ charge configuration with a perpendicular magnetic field applied. (b) Six-electron charge stability diagram around the $(1,4,1)$ ground state, as a function of $V_{m}$ and $V_{d}$. 
indicated. We describe this system using a simple Hubbardlike Hamiltonian [16,31,45],

$$
H=\sum_{i=1}^{3}\left(H_{1}^{(i)}-V_{i} n_{i}\right)+\sum_{\langle i, j\rangle} U_{c} n_{i} n_{j}-\sum_{\langle i, j\rangle, \eta} \frac{t_{i j}}{\sqrt{2}} c_{i \eta}^{\dagger} c_{j \eta},
$$

where $n_{i}=\sum_{\eta} c_{i \eta}^{\dagger} c_{i \eta}$ is the number operator for $\operatorname{dot} i, c_{i \eta}$ annihilates an electron on dot $i$ with spin $\eta, U_{c}$ accounts for the cross capacitance between neighboring dots, and $H_{1}^{(i)}$ is the single-dot many-particle Hamiltonian for dot $i$ as described above. We thus made several simplifying assumptions: (i) The gate-induced potentials are smooth enough so that they affect all electronic orbitals in the same way. (ii) The separation between the dots is large enough to allow us to treat the interdot electrostatic energy as being dependent only on the $n_{i}$ and not on the exact orbital configuration of the electrons on the neighboring dots. (iii) All tunneling processes we will consider below mostly involve a $(0,0)$ orbital on a lateral dot and a $(0, \pm 1)$ orbital on the central dot; since all $(0, \pm 1)$ orbitals have the same radial structure, we assume that this allows us to use tunneling coefficients $t_{i j}$ that are independent of the exact electronic orbitals involved.

We first study the electrostatic properties of $H$ by diagonalizing the first two terms in Eq. (6). The charge stability diagram in Fig. 2(b) shows the resulting six-electron groundstate charge configuration $\left(n_{1}, n_{2}, n_{3}\right)$, where $n_{i}$ is the number of electrons on dot $i$, as a function of the detuning parameters $V_{d}=\frac{1}{2}\left(V_{3}-V_{1}\right)$ and $V_{m}=\frac{1}{2}\left(V_{1}+V_{3}\right)-V_{2}$. We fixed $V_{1}+$ $4 V_{2}+V_{3}$ and focused on the regime around the $(1,4,1)$ state. As indicated in Fig. 2(a), we assumed different dot sizes, $\sigma_{0}=30 \mathrm{~nm}$ for the central dot and $\sigma_{0}=20 \mathrm{~nm}$ for the lateral dots, which results in a good ratio between the orbital splitting on the outer dots and the splitting of the many-electron states in the middle dot [46]. Furthermore, we used $U_{c}=0.2 \omega_{0}$ (where $\omega_{0}$ is the bare level splitting on the central dot) and set $\omega_{c} / \omega_{0}=0.1, \kappa=0.5$, and $m^{*} / m_{e}=0.067$.

In the $(1,4,1)$ region, the four lowest-energy six-particle states with $S^{2}=0$ can be written as

$$
\begin{gathered}
|0\rangle=\left|S_{\alpha} S_{(13)}\right\rangle, \\
|1\rangle=\frac{1}{\sqrt{3}}\left[\left|T_{\beta}^{0} T_{(13)}^{0}\right\rangle-\left|T_{\beta}^{-} T_{(13)}^{+}\right\rangle-\left|T_{\beta}^{+} T_{(13)}^{-}\right\rangle\right], \\
|2\rangle=\left|S_{\beta} S_{(13)}\right\rangle, \\
|3\rangle=\left|S_{\gamma} S_{(13)}\right\rangle,
\end{gathered}
$$

where $\left|S_{(13)}\right\rangle$ and $\left|T_{(13)}\right\rangle$ indicate pairing in a singlet or triplet state of the two electrons in the outer dots, and $\left|S_{\alpha, \beta, \gamma}\right\rangle$ and $\left|T_{\beta}\right\rangle$ are the lowest four-particle singlets and triplet on the central dot, see above.

The qubit. We propose to tune close to the degeneracy of $\left|S_{\alpha}\right\rangle$ and $\left|T_{\beta}\right\rangle$ on the central dot, which for $\sigma_{0}=30 \mathrm{~nm}$ happens at $B \approx 75 \mathrm{mT}$. The two lowest-energy singlet states $|0\rangle$ and $|1\rangle$ can then be used as qubit basis, and the singlets $|2\rangle$ and $|3\rangle$ will be split off by an energy much larger than the qubit splitting.

We assume that $t / \Delta \ll 1$, with $t$ the magnitude of the tunnel couplings (typically $t \sim 10 \mu \mathrm{eV}$ ) and $2 \Delta$ the width of the $(1,4,1)$ region; see its definition in Fig. 2(b). Then we can treat the tunnel coupling perturbatively for most of the $(1,4,1)$ region, and we thus project the full Hamiltonian (6) onto the qubit subspace by means of a Schrieffer-Wolff transformation [37], yielding to order $t^{2}$

$$
H_{\mathrm{qb}}=\frac{1}{2}\left(E_{S T}+J_{z}\right) \sigma_{z}+J_{x} \sigma_{x},
$$

where $\sigma_{x, z}$ are Pauli matrices. The qubit splitting is dominated by the singlet-triplet splitting on the central dot $E_{S T}=E_{T_{\beta}^{0}}-$ $E_{S_{\alpha}}$ [see Fig. 1(b)], which follows to good approximation from the expressions given in Eq. (5),

$$
E_{S T} \approx \gamma_{0} \omega_{0}+\omega_{c}
$$

with $\gamma_{0}=-0.235 \kappa+0.128 \kappa^{2}$, accurate for $\kappa \lesssim 0.5$ (see Ref. [37] for all derivations and an explicit expression for $\left.\gamma_{0}\right)$. We wrote $E_{S T}$ here up to linear order in $\omega_{c} / \omega_{0}$; the next correction is smaller by a factor $\approx 10^{-2} \kappa \omega_{c} / \omega_{0}$. Through $\omega_{c} \propto B$, this term, and thus the qubit splitting, can be easily tuned over tens of $\mu \mathrm{eV}$. We emphasize that this magnetic field dependence arises through coupling of the field to the orbital degrees of freedom of the electrons; the (singlet-only) qubit subspace is insensitive to the coupling of magnetic fields to the spin of the electrons.

Close to the line where $V_{d}=0$ and assuming approximately symmetric tunnel couplings $t_{12} \approx t_{23}$, the two exchange terms read as [37]

$$
\begin{gathered}
J_{z} \approx-t^{2}\left[\frac{\Delta}{\Delta^{2}-V_{m}^{2}}+\frac{3\left(\Delta+\omega_{c}\right)}{\left(\Delta+\omega_{c}\right)^{2}-V_{m}^{2}}\right], \\
J_{x} \approx \frac{\sqrt{6} t \Delta}{\Delta^{2}-V_{m}^{2}}\left[\delta t+\frac{2 t V_{m}}{\Delta^{2}-V_{m}^{2}} V_{d}\right]
\end{gathered}
$$

for $\Delta$ as defined in Fig. 2(b) and with $t=\frac{1}{2}\left(t_{12}+t_{23}\right)$ and $\delta t=t_{12}-t_{23}$. We see that $J_{z}$ in general presents a small tuning-dependent correction to the qubit splitting, which is dominated by $E_{S T}$, whereas $J_{x}$ provides a coupling to $\sigma_{x}$ linear in $\delta t$ and/or $V_{d}$ (depending on tuning), which can be used to drive Rabi oscillations.

We now discuss two regimes of special interest in the charge stability diagram shown in Fig. 2(b): (i) In the resonant-exchange $(\mathrm{RX})$ regime, close to the top and bottom of the $(1,4,1)$ region, the strong coupling to the other charge states offers fast qubit control through $V_{d}$ [8]. In Fig. 3(a), we show the lowest-lying states as a function of $V_{d}$ along the horizontal dashed line in Fig. 2(b) $\left(V_{m} / \omega_{0}=0.27\right)$ calculated from the Hamiltonian as given in (6), where we ignored the Zeeman splitting for clarity. We used the same parameters as in Fig. 2(b) and further set $t=25 \mu \mathrm{eV}$ and $\delta t=0$. We labeled the two qubit states $|0\rangle$ and $|1\rangle$, three spin triplets $\left|T_{1,2,3}\right\rangle$, and a spin quintuplet $|Q\rangle$; including the Zeeman effect, a triplet (quintuplet) acquires an additional threefold (fivefold) splitting of $1.7 \mu \mathrm{eV}$ for $\omega_{c} / \omega_{0}=0.1$. (ii) In the center of the $(1,4,1)$ region, we find a SS where the qubit is to linear order insensitive to fluctuations of the potentials $V_{i}$, offering some protection against charge noise. In Fig. 3(b), we show the spectrum at the SS for the same parameters as in Fig. 3(a), now as a function of $\delta t$ while setting $V_{d}=0$. At the SS exchange effects are much smaller and thus the qubit splitting is closer to $E_{S T}\left(\approx 18.3 \mu \mathrm{eV}\right.$ for $\left.\omega_{c} / \omega_{0}=0.1\right)$, but apart from that the spectrum looks similar to the RX regime. 

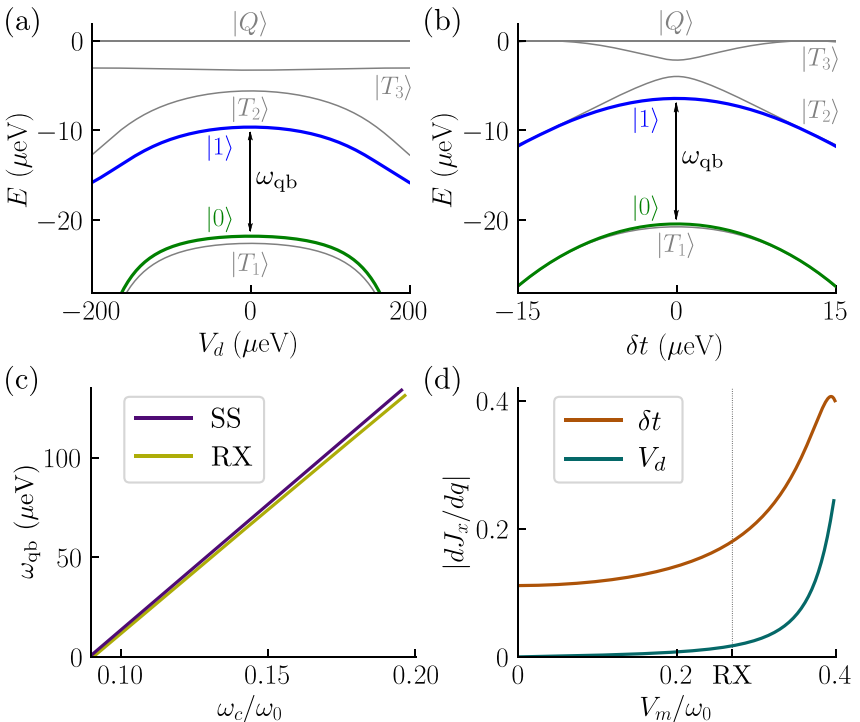

FIG. 3. Low-energy part of the spectrum of the Hamiltonian (6) (a) as a function of $V_{d}$ at $V_{m} / \omega_{0}=0.27$ and (b) as a function of $\delta t$ at the SS, $V_{d}=V_{m}=0$. The green and blue lines show the spin-singlet qubit states $|0\rangle$ and $|1\rangle$ respectively; the gray lines show the spin triplet and quintuplet states. (c) The qubit splitting as a function of the magnetic field, where $\omega_{c} / \omega_{0}=0.1$ corresponds to $B \approx 75 \mathrm{mT}$. (d) The derivative $d J_{x} / d q$ for $q \in\left\{\delta t, V_{d}\right\}$ as a function of $V_{m}$ and at $V_{d}=0$.

In Fig. 3(c), we plot the qubit splitting $\omega_{\mathrm{qb}}$ as a function of the magnetic field, in the RX regime $\left(V_{m}=0.27, V_{d}=0\right.$, yellow line) and at the SS (purple line). This confirms the high degree of tunability of our qubit. We further note how the spectra in Figs. 3(a) and 3(b) strongly resemble those in the XOSO spin-qubit proposals of Refs. [31,32], the main difference being the large and straightforwardly tunable qubit splitting $\omega_{\mathrm{qb}} \propto B$ in our proposal. This permits an efficient and adaptable coupling to other systems such as microwave cavities which can be used to couple distant qubits [47-50].

Qubit operation. Single-qubit rotations can be performed via resonant Rabi driving, using a sinusoidal modulation of a tuning parameter $q=\left\{V_{d}, V_{m}, t, \delta t\right\}$ with a small amplitude $\tilde{q}$ and frequency $\omega$, i.e., $q(t)=q_{0}+\tilde{q} \sin (\omega t)$. For small enough $\tilde{q}$, the qubit Hamiltonian (11) can be approximated as

$$
H_{\mathrm{qb}}=\frac{1}{2} \omega_{\mathrm{qb}} \sigma_{z}+A_{q} \sin (\omega t) \sigma_{x},
$$

where $A_{q}=\tilde{q}\left(d J_{x} / d q\right)_{q=q_{0}}$. Driving the qubit resonantly, $\omega=\omega_{\mathrm{qb}}$, then induces Rabi oscillations with a frequency $A_{q}$. At the RX regime, where we can use $V_{d}$ as the driving parameter, an amplitude of $\tilde{V}_{d}=5-10 \mu \mathrm{eV}$ gives a Rabi period of $T_{\text {Rabi }} \approx 20-40 \mathrm{~ns}$. At the SS, Rabi rotations are much more efficient via a driving of $\delta t$, which gives a period of $T_{\mathrm{Rabi}} \approx$ $20 \mathrm{~ns}$ for an amplitude $\delta \tilde{t}=2 \mu \mathrm{eV}$. Fast qubit rotations can therefore be achieved both in the RX regime and at the SS. In Fig. 3(d), we plot the "efficiency" $d J_{x} / d q$ of the two driving parameters $q \in\left\{\delta t, V_{d}\right\}$ as a function of $V_{m}$, along the line $V_{d, 0}=0$. We see that at the SS the sensitivity to $V_{d}$ vanishes, in accordance with Eq. (14), whereas driving of $\delta t$ stays effective all the way down to $V_{m}=0$.
Qubit initialization and readout can be accomplished by standard spin-to-charge conversion, i.e., pulsing the qubit to one of the neighboring charge configurations that has only one low-lying six-particle singlet state. For example, when tuning into the $(1,3,2) /(2,3,1)$ charge regions, only the qubit state $|0\rangle$ is adiabatically connected to the new groundstate charge configuration. This allows for initialization in $|0\rangle$ as well as readout of the qubit by means of charge detection.

Decoherence. In most GaAs-based spin qubits, the main source of decoherence is the fluctuating bath of nuclear spins that couples to the electron spins via contact hyperfine interaction. On a mean-field level, the effect of this interaction can be described by the Hamiltonian $H_{\mathrm{hf}}=\frac{1}{2} g \mu_{\mathrm{B}} \sum_{i} \mathbf{K}_{i} \cdot \boldsymbol{\sigma}_{i}$, with $\mathbf{K}_{i}$ being a random effective nuclear field acting on electron $i$, typically of the order of a few $\mathrm{mT}$. In the device we propose in this paper, both qubit states are singlets and therefore the qubit splitting is not directly influenced by any intrinsic or external (gradient) of Zeeman fields acting on the electrons, thereby reducing the hyperfine-induced decoherence dramatically $[31,32]$. We estimate the coupling between the nuclear magnetic moments and the orbital degrees of freedom of the electrons to be negligible and dominated by hyperfine coupling of the qubit states to nearby triplet states, which leads to random higher order shifts of the qubit levels [31]. The timescale of this residual hyperfine-induced dephasing can be estimated as $T_{2}^{*} \sim A_{q} \hbar(\delta \varepsilon)^{2} / \sigma_{K}^{4}$, where $\delta \varepsilon$ is the energy splitting between $|0\rangle$ and $\left|T_{1}\right\rangle$; see Figs. 3(a) and 3(b) [37]. For the range of parameters considered here, we find $T_{2}^{*} \sim$ 0.5-5 $\mu$ s [37], giving a number of visible, coherent Rabi oscillations of $n_{\text {coh }}=T_{2}^{*} / T_{\text {Rabi }} \sim 25-250$.

Another source of decoherence for exchange-based qubits are low-frequency fluctuations in the electrostatic environment of the system. A common way to mitigate such charge noise is to operate the qubit at the SS [Fig. 3(b)], where the qubit splitting is insensitive to fluctuations in the potentials $V_{i}$ to leading order; there we find a dephasing time of $T_{2}^{*} \gg$ $10 \mu \mathrm{s}$. Away from the SS, the effects of charge noise are larger. At the RX regime [Fig. 3(a)], far away from the SS, the contribution from charge noise to dephasing becomes similar to that of nuclear noise, with a dephasing time of $T_{2}^{*} \sim 0.5 \mu \mathrm{s}$ [37].

Finally, qubit relaxation via electron-phonon coupling causes qubit decoherence. The relaxation rate can be estimated using Fermi's golden rule and depends on the qubit splitting and on the strength of the exchange interaction [31]. In the RX regime, where the qubit splitting can be extensively tuned through $\omega_{c}$, we estimate relaxation rates from $\Gamma_{\text {rel }} \sim$ $1 \mathrm{GHz}$ for $\omega_{\mathrm{qb}} \sim 50 \mu \mathrm{eV}$ to $\Gamma_{\text {rel }} \sim 1 \mathrm{MHz}$ for $\omega_{\mathrm{qb}} \sim 10 \mu \mathrm{eV}$. And, as is common in exchange-based qubits [18,31], the relaxation rate is strongly suppressed as we approach the SS.

Conclusions. We propose a six-electron exchange-only singlet-only spin qubit hosted in a GaAs linear triple quantum dot. Its singlet-only nature makes the qubit intrinsically insensitive to randomly fluctuating nuclear fields. The qubit can be operated fully electrically, either in an RX regime which enables fast qubit operations or at a SS where the qubit is better protected against charge noise. Furthermore, the fact that the qubit splitting is highly tunable over a large range of energies allows for efficient and adaptable coupling 
to microwave resonators, enabling coupling of distant qubits. The only ingredient on which this tunability relies is the appearance of a ground-state singlet-triplet transition at finite magnetic field in the multiparticle spectrum of the central dot. This is a very commonly observed feature in quantum dots of various shapes and sizes.
Acknowledgments. This work is part of FRIPRO Project No. 274853, which is funded by the Research Council of Norway (RCN), and was also partly supported by the Centers of Excellence funding scheme of the RCN, Project No. 262633, QuSpin.

A.S. and J.H.Q. contributed equally to this work.
[1] R. Hanson, L. P. Kouwenhoven, J. R. Petta, S. Tarucha, and L. M. K. Vandersypen, Rev. Mod. Phys. 79, 1217 (2007).

[2] F. A. Zwanenburg, A. S. Dzurak, A. Morello, M. Y. Simmons, L. C. L. Hollenberg, G. Klimeck, S. Rogge, S. N. Coppersmith, and M. A. Eriksson, Rev. Mod. Phys. 85, 961 (2013).

[3] D. P. DiVincenzo, D. Bacon, J. Kempe, G. Burkard, and K. B. Whaley, Nature (London) 408, 339 (2000).

[4] M. Russ and G. Burkard, J. Phys.: Condens. Matter 29, 393001 (2017).

[5] E. A. Laird, J. M. Taylor, D. P. DiVincenzo, C. M. Marcus, M. P. Hanson, and A. C. Gossard, Phys. Rev. B 82, 075403 (2010).

[6] L. Gaudreau, G. Granger, A. Kam, G. C. Aers, S. A. Studenikin, P. Zawadzki, M. Pioro-Ladrière, Z. R. Wasilewski, and A. S. Sachrajda, Nat. Phys. 8, 54 (2011).

[7] J. Medford, J. Beil, J. M. Taylor, S. D. Bartlett, A. C. Doherty, E. I. Rashba, D. P. DiVincenzo, H. Lu, A. C. Gossard, and C. M. Marcus, Nat. Nanotechnol. 8, 654 (2013).

[8] J. Medford, J. Beil, J. M. Taylor, E. I. Rashba, H. Lu, A. C. Gossard, and C. M. Marcus, Phys. Rev. Lett. 111, 050501 (2013).

[9] K. Eng, T. D. Ladd, A. Smith, M. G. Borselli, A. A. Kiselev, B. H. Fong, K. S. Holabird, T. M. Hazard, B. Huang, P. W. Deelman, I. Milosavljevic, A. E. Schmitz, R. S. Ross, M. F. Gyure, and A. T. Hunter, Sci. Adv. 1, e1500214 (2015).

[10] R. W. Andrews, C. Jones, M. D. Reed, A. M. Jones, S. D. Ha, M. P. Jura, J. Kerckhoff, M. Levendorf, S. Meenehan, S. T. Merkel, A. Smith, B. Sun, A. J. Weinstein, M. T. Rakher, T. D. Ladd, and M. G. Borselli, Nat. Nanotechnol. 14, 747 (2019).

[11] J.-T. Hung, J. Fei, M. Friesen, and X. Hu, Phys. Rev. B 90, 045308 (2014).

[12] C. G. Péterfalvi and G. Burkard, Phys. Rev. B 96, 245412 (2017).

[13] M. Russ and G. Burkard, Phys. Rev. B 91, 235411 (2015).

[14] F. Martins, F. K. Malinowski, P. D. Nissen, E. Barnes, S. Fallahi, G. C. Gardner, M. J. Manfra, C. M. Marcus, and F. Kuemmeth, Phys. Rev. Lett. 116, 116801 (2016).

[15] J. Yoneda, K. Takeda, T. Otsuka, T. Nakajima, M. R. Delbecq, G. Allison, T. Honda, T. Kodera, S. Oda, Y. Hoshi, N. Usami, K. M. Itoh, and S. Tarucha, Nat. Nanotechnol. 13, 102 (2018).

[16] J. M. Taylor, V. Srinivasa, and J. Medford, Phys. Rev. Lett. 111, 050502 (2013).

[17] C. Zhang, X. C. Yang, and X. Wang, Phys. Rev. A 97, 042326 (2018).

[18] A. Sala and J. Danon, Phys. Rev. B 98, 245409 (2018).

[19] V. N. Golovach, A. Khaetskii, and D. Loss, Phys. Rev. Lett. 93, 016601 (2004).

[20] M. Raith, P. Stano, F. Baruffa, and J. Fabian, Phys. Rev. Lett. 108, 246602 (2012).
[21] M. Raith, P. Stano, and J. Fabian, Phys. Rev. B 86, 205321 (2012).

[22] A. Hofmann, V. F. Maisi, T. Krähenmann, C. Reichl, W. Wegscheider, K. Ensslin, and T. Ihn, Phys. Rev. Lett. 119, 176807 (2017).

[23] V. Srinivasa, J. M. Taylor, and C. Tahan, Phys. Rev. B 94, 205421 (2016).

[24] A. J. Landig, J. V. Koski, P. Scarlino, U. C. Mendes, A. Blais, C. Reichl, W. Wegscheider, A. Wallraff, K. Ensslin, and T. Ihn, Nature (London) 560, 179 (2018).

[25] S. P. Harvey, C. G. L. Bøttcher, L. A. Orona, S. D. Bartlett, A. C. Doherty, and A. Yacoby, Phys. Rev. B 97, 235409 (2018).

[26] M. D. Reed, B. M. Maune, R. W. Andrews, M. G. Borselli, K. Eng, M. P. Jura, A. A. Kiselev, T. D. Ladd, S. T. Merkel, I. Milosavljevic, E. J. Pritchett, M. T. Rakher, R. S. Ross, A. E. Schmitz, A. Smith, J. A. Wright, M. F. Gyure, and A. T. Hunter, Phys. Rev. Lett. 116, 110402 (2016).

[27] Y.-P. Shim and C. Tahan, Phys. Rev. B 93, 121410(R) (2016).

[28] F. K. Malinowski, F. Martins, P. D. Nissen, S. Fallahi, G. C. Gardner, M. J. Manfra, C. M. Marcus, and F. Kuemmeth, Phys. Rev. B 96, 045443 (2017).

[29] D. Bacon, J. Kempe, D. A. Lidar, and K. B. Whaley, Phys. Rev. Lett. 85, 1758 (2000).

[30] M. Friesen, J. Ghosh, M. A. Eriksson, and S. N. Coppersmith, Nat. Commun. 8, 15923 (2017).

[31] A. Sala and J. Danon, Phys. Rev. B 95, 241303(R) (2017).

[32] M. Russ, J. R. Petta, and G. Burkard, Phys. Rev. Lett. 121, 177701 (2018).

[33] D. Culcer, X. Hu, and S. Das Sarma, Phys. Rev. B 82, 205315 (2010).

[34] M. Eto, J. Phys. Soc. Jpn. 66, 2244 (1997).

[35] An in-plane magnetic field would also induce crossings between singlet and triplet many-particle states, but due to the strong confinement of the electrons along one of the axes perpendicular to the field, a much larger magnetic field would be required to achieve the same effect.

[36] M. Rontani, F. Rossi, F. Manghi, and E. Molinari, Phys. Rev. B 59, 10165 (1999).

[37] See Supplemental Material at http://link.aps.org/supplemental/ 10.1103/PhysRevResearch.2.012062 which includes Refs. [38-43], for more detailed discussions and derivations of the equations presented in the main text.

[38] V. N. Golovach, A. Khaetskii, and D. Loss, Phys. Rev. B 77, 045328 (2008).

[39] L. P. Kouwenhoven, T. H. Oosterkamp, M. W. S. Danoesastro, M. Eto, D. G. Austing, T. Honda, and S. Tarucha, Science 278, 1788 (1997).

[40] M. Rontani, Electronic states in semiconductor quantum dots, Ph.D. thesis, Università degli Studi di Modena e Reggio Emilia, Modena, Italy, 1999. 
[41] F. C. Zhang and S. Das Sarma, Phys. Rev. B 33, 2903 (1986).

[42] L. Jacak, J. Krasnyj, D. Jacak, W. Salejda, and A. Mituś, Acta Phys. Pol. A 99, 277 (2001).

[43] V. Srinivasa, H. Xu, and J. M. Taylor, Phys. Rev. Lett. 114, 226803 (2015).

[44] S. M. Reimann and M. Manninen, Rev. Mod. Phys. 74, 1283 (2002).

[45] G. Burkard, D. Loss, and D. P. DiVincenzo, Phys. Rev. B 59, 2070 (1999).

[46] The dependence of the orbital spacing on the dot size allows us to design a device with an orbital splitting on the outer dots larger than the level splittings in the central multielectron dot, which enables full spin blockade in the regime where the qubit is operated.

[47] M. Russ and G. Burkard, Phys. Rev. B 92, 205412 (2015).

[48] M. Russ, F. Ginzel, and G. Burkard, Phys. Rev. B 94, 165411 (2016).

[49] A. J. Landig, J. V. Koski, P. Scarlino, C. Müller, J. C. Abadillo-Uriel, B. Kratochwil, C. Reichl, W. Wegscheider, S. N. Coppersmith, M. Friesen, A. Wallraff, T. Ihn, and K. Ensslin, Nat. Commun. 10, 5037 (2019).

[50] G. Burkard, M. J. Gullans, X. Mi, and J. R. Petta, Nat. Rev. Phys. 2, 129 (2020). 